DAMTP/96-28

\title{
CROSSED PRODUCTS BY A COALGEBRA
}

\author{
Tomasz Brzeziński円 \\ Department of Applied Mathematics \& Theoretical Physics \\ University of Cambridge, Cambridge CB3 9EW
}

March 1996

\begin{abstract}
We introduce the notion of a crossed product of an algebra by a coalgebra $C$, which generalises the notion of a crossed product by a bialgebra wellstudied in the theory of Hopf algebras. The result of such a crossed product is an algebra which is also a right $C$-comodule. We find the necessary and sufficient conditions for two coalgebra crossed products be equivalent. We show that the two-dimensional quantum Euclidean group is a coalgebra crossed product. The paper is completed with an appendix describing the dualisation of construction of coalgebra crossed products.
\end{abstract}

\section{Introduction}

The notion of a crossed product by a bialgebra was first introduced in the context of cohomology of algebras over a bialgebra [15]. It was then generalised and throughly studied in the relation to the Hopf-Galois theory of non-commutative rings and invariant theory of algebraic groups [7] [6] [1] [2]. With the emergence of quantum groups the theory of Hopf-Galois extensions and crossed products by Hopf algebras became interesting also from geometric point of view (cf. [14 and references therein). Hopf-Galois extensions are now understood as quantum group principal bundles [4] [12] and a certain kind of a crossed product, known as a cleft extension, corresponds to a trivial quantum group principal bundle. In search for a suitable quantum group gauge theory on quantum homogeneous spaces we have recently proposed [5] a generalisation of quantum group principal bundles in which the structure quantum group is replaced by a coalgebra. We also introduced the notion of a trivial coalgebra principal bundle. As a vector space, the total space of such a bundle is isomorphic to a tensor product of a base manifold algebra and a structure coalgebra. It is therefore natural to expect that as an algebra the total space is a certain kind of a cleft extension of an algebra by a coalgebra. This leads naturally to the notion of a crossed product by a coalgebra. In this paper we give definition and study some properties of such crossed products.

\footnotetext{
${ }^{1}$ Research supported by the EPSRC grant GR/K02244
} 
Throughout the paper, all vector spaces are over a field $k$ of generic characteristic. If not stated otherwise, by an algebra we mean a unital associative algebra over $k$. The unit in an algebra is denoted by 1 . In a coalgebra $C, \Delta$ denotes the coproduct and $\epsilon: C \rightarrow k$ denotes the counit. We use the Sweedler notation to denote the coproduct in $C, \Delta c=c_{(1)} \otimes c_{(2)}$ (summation understood), for any $c \in C$. By convolution product we mean a product $*$ in a space of linear maps $C \rightarrow P$, where $P$ is an algebra, given by $f * g(c)=f\left(c_{(1)}\right) g\left(c_{(2)}\right)$. A map $C \rightarrow P$ is said to be convolution invertible if it is invertible with respect to this product.

\section{Crossed Products by a Coalgebra}

We first recall the definition of an entwining structure from [5] which forms a backbone of theory of coalgebra principal bundles and, subsequently, coalgebra crossed products. We say that a coalgebra $C$ and an algebra $P$ are entwined if there is a map $\psi: C \otimes P \rightarrow P \otimes C$ such that

$$
\begin{gathered}
\psi \circ\left(\operatorname{id}_{C} \otimes \mu\right)=\left(\mu \otimes \operatorname{id}_{C}\right) \circ \psi_{23} \circ \psi_{12}, \quad \psi(c \otimes 1)=1 \otimes c, \quad \forall c \in C \\
\left(\operatorname{id}_{P} \otimes \Delta\right) \circ \psi=\psi_{12} \circ \psi_{23} \circ\left(\Delta \otimes \operatorname{id}_{P}\right), \quad\left(\operatorname{id}_{P} \otimes \epsilon\right) \circ \psi=\epsilon \otimes \operatorname{id}_{P},
\end{gathered}
$$

where $\mu$ denotes multiplication in $P$, and $\psi_{12}=\psi \otimes \mathrm{id}_{P}$ and $\psi_{23}=\mathrm{id}_{P} \otimes \psi$. We denote the action of $\psi$ on $c \otimes u \in C \otimes P$ by $\psi(c \otimes u)=u_{\alpha} \otimes c^{\alpha}$ (summation understood).

Furthermore we assume that there is a group-like $e \in C$, i.e. $\Delta e=e \otimes e, \epsilon(e)=1$ and a map $\psi^{C}: C \otimes C \rightarrow C \otimes C$ such that for any $c \in C$

$$
(\mathrm{id} \otimes \Delta) \circ \psi^{C}=\psi_{12}^{C} \circ \psi_{23}^{C} \circ(\Delta \otimes \mathrm{id}), \quad(\mathrm{id} \otimes \epsilon) \circ \psi^{C}=\epsilon \otimes \mathrm{id}, \quad \psi^{C}(e \otimes c)=\Delta c
$$

where $\psi_{12}^{C}=\psi^{C} \otimes \operatorname{id}_{C}$ and $\psi_{23}=\operatorname{id}_{C} \otimes \psi^{C}$. We denote the action of $\psi^{C}$ on $b \otimes c$ by $\psi(b \otimes c)=$ $c_{A} \otimes b^{A}$ (summation understood).

With these assumptions $P$ is a right $C$-comodule with a coaction $\Delta_{R} u=\psi(e \otimes u)$. Moreover the fixed point subspace $M=P_{e}^{c o C}=\left\{x \in P \mid \Delta_{R} x=x \otimes e\right\}$ is a subalgebra of $P$. We call $\left(P, C, \psi, e, \psi^{C}\right)$ the entwining data. A number of examples of entwining data may be found in [5]. The main object of studies of this paper is contained in the following:

Proposition 2.1 Let $\left(P, C, \psi, e, \psi^{C}\right)$ be entwining data and let $M=P_{e}^{c o C}$. Assume that there are linear maps $\sigma: C \otimes C \rightarrow M$ and $\rho: C \otimes P \rightarrow P$ such that for all $x, y \in M, c \in C$ :

(i) $\rho(e, x)=x, \quad \rho(c, 1)=\epsilon(c)$;

(ii) $\rho\left(c_{(1)}, x_{\alpha}\right) \otimes c_{(2)}{ }^{\alpha} \in M \otimes C$;

(iii) $\rho(c, x y)=\rho\left(c_{(1)}, x_{\alpha}\right) \rho\left(c_{(2)}^{\alpha}, y\right)$;

(iv) $\sigma(e, c)=\epsilon(c), \quad \sigma\left(c_{(1)}, e_{A}\right) \otimes c_{(2)}{ }^{A}=1 \otimes c$. 
Define a linear map $M \otimes C \otimes M \otimes C \rightarrow M \otimes C$, denoted by juxtaposition, by

$$
(x \otimes b)(y \otimes c)=x \rho\left(b_{(1)}, y_{\alpha}\right) \sigma\left(b_{(2)}{ }^{\alpha}{ }_{(1)}, c_{A}\right) \otimes{b_{(2)}}^{\alpha}{ }_{(2)}{ }^{A} .
$$

Then for any $x \in M$ and $c \in C(1 \otimes e)(x \otimes c)=(x \otimes c)(1 \otimes e)=x \otimes c$. The vector space $M \otimes C$ is an associative algebra with product (因) if and only if

$$
\rho\left(a_{(1)}, \sigma\left(b_{(1)}, c_{A}\right)_{\alpha}\right) \sigma\left(a_{(2)}{ }^{\alpha}{ }_{(1)}, b_{(2)}{ }_{B}^{A}\right) \otimes a_{(2)}{ }^{\alpha}{ }_{(2)}{ }^{B}=\sigma\left(a_{(1)}, b_{A}\right) \sigma\left(a_{(2)}{ }_{(1)}^{A}, c_{B}\right) \otimes a_{(2)}{ }_{(2)}{ }^{B}
$$

and

$$
\rho\left(a_{(1)}, \rho\left(b_{(1)}, x_{\alpha}\right)_{\beta}\right) \sigma\left(a_{(2)}{ }^{\beta}{ }_{(1)}, b_{(2)}{ }_{A}^{\alpha}\right) \otimes a_{(2)}{ }^{\beta}{ }_{(2)}{ }^{A}=\sigma\left(a_{(1)}, b_{A}\right) \rho\left(a_{(2)}{ }_{(1)}, x_{\alpha}\right) \otimes a_{(2)}{ }_{(2)}{ }^{\alpha},
$$

for any $a, b, c \in C$ and $x \in M$. This algebra is denoted by $M \rtimes_{\rho, \sigma} C$ and called a crossed product by a coalgebra $C$. The pair $(\rho, \sigma)$ is called the crossed product data for the entwining data $\left(P, C, \psi, e, \psi^{C}\right)$.

Proof. It is clear from assumption (ii) that for all $b, c \in C$ and $x, y \in M,(x \otimes b)(y \otimes c) \in$ $M \otimes C$. Next we have

$$
\begin{aligned}
(1 \otimes e)(x \otimes c) & =\rho\left(e, x_{\alpha}\right) \sigma\left(e^{\alpha}{ }_{(1)}, c_{A}\right) \otimes e^{\alpha}{ }_{(2)}^{A} \stackrel{(i)}{=} x_{\alpha} \sigma\left(e^{\alpha}{ }_{(1)}, c_{A}\right) \otimes e^{\alpha}{ }_{(2)}^{A} \\
& =x \sigma\left(e, c_{A}\right) \otimes e^{A} \stackrel{(i v)}{=} x \epsilon\left(c_{A}\right) \otimes e^{A} \stackrel{\text { 这 }}{=} x \otimes c .
\end{aligned}
$$

To derive the third equality we used the fact that $x \in M=P_{e}^{c o C}$. Furthermore

$$
(x \otimes c)(1 \otimes e)=x \rho\left(c_{(1)}, 1\right) \sigma\left(c_{(2)}, e_{A}\right) \otimes c_{(3)} \stackrel{A}{\stackrel{(i)}{=}} x \sigma\left(c_{(1)}, e_{A}\right) \otimes c_{(2)} \stackrel{A}{A} \stackrel{(i v)}{=} x \otimes c .
$$

Now assume that (5) and (6) hold. Then we have

$$
\begin{aligned}
& (z \otimes a)[(x \otimes b)(y \otimes c)] \\
& =\quad z \rho\left(a_{(1)},\left(x \rho\left(b_{(1)}, y_{\alpha}\right) \sigma\left(b_{(2)}{ }^{\alpha}{ }_{(1)}, c_{A}\right)\right)_{\beta}\right) \sigma\left(a_{(2)}{ }^{\beta}{ }_{(1)}, b_{(2)}{ }^{\alpha}{ }_{(2)}{ }_{B}^{A}\right) \otimes a_{(2)}{ }^{\beta}{ }_{(2)}{ }^{B} \\
& \text { 国, } \stackrel{\text { (iii) }}{=} z \rho\left(a_{(1)}, x_{\beta \gamma}\right) \rho\left(a_{(2)}{ }^{\gamma}{ }_{(1)}, \rho\left(b_{(1)}, y_{\alpha}\right)_{\delta \mu}\right) \rho\left(a_{(2)}{ }^{\gamma}{ }_{(2)}{ }^{\mu}, \sigma\left(b_{(2)}{ }^{\alpha}{ }_{(1)}, c_{A}\right)_{\nu}\right) \times \\
& \times \sigma\left(a_{(3)}{ }^{\beta \delta \nu}{ }_{(1)}, b_{(2)}{ }^{\alpha}{ }_{(2)}{ }_{B}^{A}\right) \otimes a_{(3)}{ }^{\beta \delta \nu}{ }_{(2)}{ }^{B}
\end{aligned}
$$

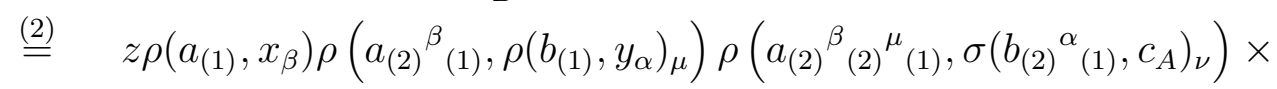

$$
\begin{aligned}
& \times \sigma\left(a_{(2)}{ }^{\beta}{ }_{(2)}^{\mu}{ }_{(2)}{ }^{\nu}{ }_{(1)}, b_{(2)}{ }^{\alpha}{ }_{(2)}{ }_{B}^{A}\right) \otimes a_{(2)}{ }^{\beta}{ }_{(2)}{ }^{\mu}{ }_{(2)}{ }^{\nu}{ }_{(2)}{ }^{B} \\
& \text { 且 } z \rho\left(a_{(1)}, x_{\beta}\right) \rho\left(a_{(2)}{ }^{\beta}{ }_{(1)}, \rho\left(b_{(1)}, y_{\alpha}\right)_{\mu}\right) \sigma\left(a_{(2)}{ }^{\beta}{ }_{(2)}{ }^{\mu}{ }_{(1)}, b_{(2)}{ }_{A}{ }_{A}\right) \times \\
& \times \sigma\left(a_{(2)}{ }^{\beta}{ }_{(2)}{ }^{\mu}{ }_{(2)}{ }^{A}{ }_{(1)}, c_{B}\right) \otimes a_{(2)}{ }^{\beta}(2)^{\mu}{ }_{(2)}{ }^{A}{ }_{(2)}{ }^{B} \\
& \text { (1) } z \rho\left(a_{(1)}, x_{\beta}\right) \sigma\left(a_{(2)}{ }^{\beta}{ }_{(1)}, b_{A}\right) \rho\left(a_{(2)}{ }^{\beta}{ }_{(2)}^{A}{ }_{(1)}, y_{\alpha}\right) \sigma\left(a_{\left.(2)^{\beta}{ }_{(2)}{ }^{A}{ }_{(2)}{ }^{\alpha}{ }_{(1)}, c_{B}\right) \otimes}\right. \\
& \otimes a_{(2)}{ }^{\beta}{ }_{(2)}{ }^{A}{ }_{(2)}{ }^{\alpha}{ }_{(2)}{ }^{B} \\
& =\left(z \rho\left(a_{(1)}, x_{\beta}\right) \sigma\left(a_{(2)}{ }^{\beta}{ }_{(1)}, b_{A}\right) \otimes a_{(2)}{ }^{\beta}{ }_{(2)}{ }^{A}\right)(y \otimes c) \\
& =[(z \otimes a)(x \otimes b)](y \otimes c) \text {. }
\end{aligned}
$$


Conversely, if we assume that (4) is an associative product in $M \otimes C$ then

$$
[(1 \otimes a)(1 \otimes b)](x \otimes c)=(1 \otimes a)[(1 \otimes b)(x \otimes c)]
$$

implies that

$$
\begin{aligned}
& \sigma\left(a_{(1)}, b_{A}\right) \rho\left(a_{(2)}{ }_{(1)}, x_{\alpha}\right) \sigma\left(a_{(2)}{ }^{A}{ }_{(2)}^{\alpha}{ }_{(1)}, c_{B}\right) \otimes a_{(2)}{ }_{(2)}{ }^{\alpha}{ }_{(2)}^{B}= \\
& \quad=\rho\left(a_{(1)}, \rho\left(b_{(1)}, x_{\alpha}\right)_{\beta} \sigma\left(b_{(2)}{ }^{\alpha}{ }_{(1)}, c_{A}\right)_{\gamma}\right) \sigma\left(a_{(2)}{ }^{\beta \gamma}{ }_{(1)}, b_{(2)}{ }_{(2)}{ }^{A}{ }_{B}\right) \otimes a_{(2)}{ }^{\beta \gamma}{ }_{(2)}{ }^{B} .
\end{aligned}
$$

Setting $x=1$ in (7) we obtain (5), while setting $c=e$ in (7) we obtain (6). This completes the proof of the proposition.

We now specify to two special cases of crossed product algebras.

Example 2.2 Let $C$ be a bialgebra $C=H$ and $P$ be a right $H$-comodule algebra. Take $e=1$, and the maps $\psi$ and $\psi^{C}$

$$
\psi(h \otimes u)=u^{(\overline{0})} \otimes h u^{(\overline{1})}, \quad \psi^{C}(h \otimes g)=g_{(1)} \otimes h g_{(2)},
$$

where $\Delta_{R} u=u^{(\overline{0})} \otimes u^{(\overline{1})}$ (summation understood). $M$ is the fixed point subalgebra of a right $H$-comodule algebra $P$, and $\psi$ restricted to $M \otimes C$ becomes the twist map, $\psi(h \otimes x)=x \otimes h$. At this point both $\psi$ and $P$ become redundant in the setting of Proposition 2.1. Conditions (i)-(iii) state that $\rho$ is a weak normalised action of $H$ on $M$, while condition (iv) specifies the normalisation of $\sigma, \sigma(1, c)=\sigma(c, 1)=\epsilon(c)$. Finally (5) states that $\sigma$ is a 2-cocycle, and (6) becomes equivalent to a twisted module condition of [7] [四]. Therefore $M \rtimes_{\rho, \sigma} H$ is a bialgebra crossed product.

Example 2.3 Let $C$ be a braided bialgebra $B$ and $P$ be a right braided $B$-comodule algebra both living in the same braided category, and $e=1$ (for the review on braided bialgebras see e.g. 90). Then the maps $\psi$ and $\psi^{C}$ are

$$
\psi(b \otimes u)=\Psi\left(b \otimes u^{(\overline{0})}\right) u^{(\overline{1})}, \quad \psi^{C}(b \otimes c)=\Psi\left(b \otimes c_{(1)}\right) c_{(2)},
$$

where $\Psi$ denotes the braiding and $\Delta_{R} u=u^{(\overline{0})} \otimes u^{(\overline{1})}$. In this case $\psi$ restricted to $B \otimes M$ coincides with $\Psi$. Conditions (i)-(iii) state now that $\rho$ is a normalised braided weak action of $B$ on $M$, while conditions (iv) fix the normalisation of $\sigma$ as in Example 2.2. Finally, using the diagrammatic technique introduced in [8], in which $\Psi=\lambda^{\prime}$, products are denoted by $Y$, and coproducts by $\lambda$, conditions (5) and (6) come out respectively:
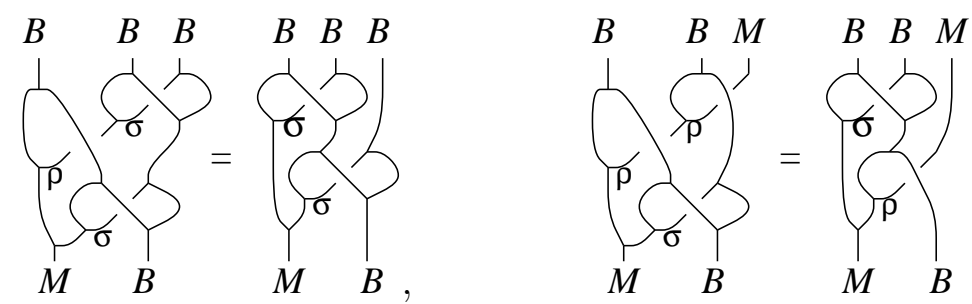
Thus we obtain the generalisation of braided crossed products introduced in [10] to the case of non-trivial $\sigma$.

Therefore the notion of a crossed product by a coalgebra in Proposition 2.1 generalises both the notion of a crossed product by a bialgebra and by a braided bialgebra. The crossed product algebra $M \rtimes_{\rho, \sigma} C$ is a left $M$-module with the natural action $x \otimes y \otimes c \mapsto x y \otimes c$. It is also a right $C$-comodule with a coaction $\Delta_{R}: x \otimes c \mapsto x \otimes c_{(1)} \otimes c_{(2)}$. Contrary to the bialgebra crossed products $M \rtimes_{\rho, \sigma} C$ need not be a right $C$-comodule algebra even if $C$ is a bialgebra. Instead, we have the following

Lemma 2.4 Let $M \rtimes_{\rho, \sigma} C$ be a crossed product as in Proposition 2.1 and let $\Delta_{R}: M \rtimes_{\rho, \sigma} C \rightarrow$ $M \rtimes_{\rho, \sigma} C \otimes C$ be a natural right coaction, $\Delta_{R}: x \otimes c \rightarrow x \otimes c_{(1)} \otimes c_{(2)}$. Then

$$
\Delta_{R}((x \otimes b)(y \otimes c))=\left(x \otimes b_{(1)}\right)\left(y_{\alpha} \otimes c_{A}\right) \otimes b_{(2)}^{\alpha A}
$$

for any $x, y \in M$ and $b, c \in C$.

Proof. We compute

$$
\begin{aligned}
\left(x \otimes b_{(1)}\right)\left(y_{\alpha} \otimes c_{A}\right) \otimes b_{(2)}{ }^{\alpha A} & =x \rho\left(b_{(1)}, y_{\alpha \beta}\right) \sigma\left(b_{(2)}{ }^{\beta}{ }_{(1)}, c_{A B}\right) \otimes b_{(2)}{ }^{\beta}{ }_{(2)}^{B} \otimes b_{(3)}{ }^{\alpha A} \\
& \underline{\underline{2}} \quad x \rho\left(b_{(1)}, y_{\alpha}\right) \sigma\left(b_{(2)}{ }^{\alpha}{ }_{(1)}, c_{A}\right) \otimes b_{(2)}{ }^{\alpha}{ }_{(2)}{ }^{A}{ }_{(1)} \otimes b_{(2)}{ }^{\alpha}{ }_{(2)}{ }_{(2)} \\
& =\Delta_{R}((x \otimes b)(y \otimes c)) .
\end{aligned}
$$

Now we give an example of a crossed product by a coalgebra coming from the theory of coalgebra bundles [5].

Example 2.5 Let P,C, $\psi, e, \psi^{C}$ and $M$ be as in Proposition 2.1. Furthermore assume that there exists a convolution invertible map $\Phi: C \rightarrow P$ such that $\Phi(e)=1$ and

$$
\psi \circ\left(\operatorname{id}_{C} \otimes \Phi\right)=\left(\Phi \otimes \operatorname{id}_{C}\right) \circ \psi^{C}
$$

Define maps $\rho: C \otimes P \rightarrow P$ and $\sigma: C \otimes C \rightarrow P$ by

$$
\rho(c, u)=\Phi\left(c_{(1)}\right) u_{\alpha} \Phi^{-1}\left(c_{(2)}^{\alpha}\right), \quad \sigma(b, c)=\Phi\left(b_{(1)}\right) \Phi\left(c_{A}\right) \Phi^{-1}\left(b_{(2)}{ }^{A}\right) .
$$

Then there is a crossed product algebra $M \rtimes_{\rho, \sigma} C$. Explicitly the product in $M \rtimes_{\rho, \sigma} C$ reads

$$
(x \otimes b)(y \otimes c)=x \Phi\left(b_{(1)}\right) y_{\alpha} \Phi\left(c_{A}\right) \Phi^{-1}\left(b_{(2)}^{\alpha A}{ }_{(1)}\right) \otimes b_{(2)}^{\alpha A}{ }_{(2)} .
$$

The algebra $M \rtimes_{\rho, \sigma} C$ is isomorphic to $P$. It is called a cleft extension of $M$ by $C$ and is denoted by $M \rtimes_{\Phi} C$. 
Proof. We first show that the output of the map $\sigma$ (9) is indeed in $M$. We compute

$$
\begin{aligned}
& \Delta_{R} \sigma(b, c) \stackrel{\text { 通 }}{=} \Phi\left(b_{(1)}\right)_{\alpha} \Phi\left(c_{A}\right)_{\beta} \Phi^{-1}\left(b_{(2)}{ }^{A}\right)_{\gamma} \otimes e^{\alpha \beta \gamma} \text { 贯) } \Phi\left(b_{(1)}\right) \Phi\left(c_{A B}\right) \Phi^{-1}\left(b_{(3)}{ }^{A}\right)_{\gamma} \otimes b_{(2)}{ }^{B \gamma} \\
& \text { 且 } \Phi\left(b_{(1)}\right) \Phi\left(c_{A}\right) \Phi^{-1}\left(b_{(2)}{ }^{A}{ }_{(2)}\right)_{\gamma} \otimes b_{(2)}{ }^{A}{ }_{(1)}^{\gamma}=\Phi\left(b_{(1)}\right) \Phi\left(c_{A}\right) \Phi^{-1}\left(b_{(2)}{ }^{A}\right) \otimes e \text {. }
\end{aligned}
$$

To derive the last equality we used the following property of $\Phi^{-1}$,

$$
\Phi^{-1}(c) \otimes e=\Phi^{-1}\left(c_{(2)}\right)_{\alpha} \otimes c_{(1)}{ }^{\alpha},
$$

which is easily obtained from (8). Therefore $\sigma$ maps $C \otimes C$ to $M$ as required.

Next we check that $\rho$ and $\sigma$ satisfy (i)-(iv) in Proposition 2.1. The condition (i) is obvious. For (ii) we take any $c \in C$ and $x \in M$ and compute

$$
\begin{aligned}
& \Delta_{R}\left(\rho\left(c_{(1)}, x_{\alpha}\right)\right) \otimes c_{(2)}{ }^{\alpha}=\psi\left(e, \Phi\left(c_{(1)}\right) x_{\alpha \beta} \Phi^{-1}\left(c_{(2)}{ }^{\beta}\right)\right) \otimes c_{(3)}{ }^{\alpha} \\
& \text { 22) } \psi\left(e, \Phi\left(c_{(1)}\right) x_{\alpha} \Phi^{-1}\left(c_{(2)}{ }^{\alpha}(1)\right)\right) \otimes c_{(2)}{ }^{\alpha}{ }_{(2)} \\
& \text { 送 } \Phi\left(c_{(1)}\right)_{\beta} x_{\alpha \gamma} \Phi^{-1}\left(c_{(2)}{ }^{\alpha}{ }_{(1)}\right)_{\delta} \otimes e^{\beta \gamma \delta} \otimes c_{(2)}{ }^{\alpha}{ }_{(2)} \\
& \underline{\underline{8}} \Phi\left(c_{(1)}\right) x_{\alpha \gamma} \Phi^{-1}\left(c_{(3)}{ }^{\alpha}(1)\right)_{\delta} \otimes c_{(2)}{ }^{\gamma \delta} \otimes c_{(3)}{ }^{\alpha}(2) \\
& \text { 221 } \Phi\left(c_{(1)}\right) x_{\alpha} \Phi^{-1}\left(c_{(2)}{ }^{\alpha}(2)\right)_{\delta} \otimes c_{(2)}{ }^{\alpha}{ }_{(1)}{ }^{\delta} \otimes c_{(2)}{ }^{\alpha}{ }_{(3)} \\
& 111 .\left(c_{(1)}\right) x_{\alpha \beta} \Phi^{-1}\left(c_{(2)}{ }^{\beta}\right) \otimes e \otimes c_{(3)}{ }^{\alpha} \text {. }
\end{aligned}
$$

To check condition (iii) we take any $c \in C, x, y \in M$ and compute

$$
\begin{aligned}
& \rho\left(c_{(1)}, x_{\alpha}\right) \rho\left(c_{(2)}{ }^{\alpha}, y\right)=\Phi\left(c_{(1)}\right) x_{\alpha \beta} \Phi^{-1}\left(c_{(2)}{ }^{\beta}\right) \Phi\left(c_{(3)}{ }^{\alpha}{ }_{(1)}\right) y_{\gamma} \Phi^{-1}\left(c_{(3)}{ }^{\alpha}(2)^{\gamma}\right) \\
& \text { 且 } \Phi\left(c_{(1)}\right) x_{\alpha} \Phi^{-1}\left(c_{(2)}{ }^{\alpha}{ }_{(1)}\right) \Phi\left(c_{(2)}{ }^{\alpha}{ }_{(2)}\right) y_{\gamma} \Phi^{-1}\left(c_{(2)}{ }^{\alpha}{ }_{(3)}{ }^{\gamma}\right)
\end{aligned}
$$

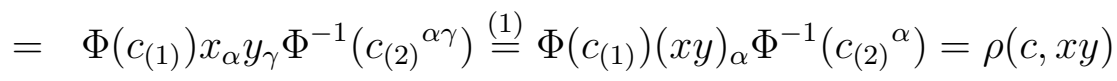

The first part of condition (iv) is obvious. For the second one we have

$$
\begin{aligned}
& \sigma\left(c_{(1)}, e_{A}\right) \otimes c_{(2)}{ }^{A}=\Phi\left(c_{(1)}\right) \Phi\left(e_{A B}\right) \Phi^{-1}\left(c_{(2)}{ }^{B}\right) \otimes c_{(3)}{ }^{A} \\
& \text { 典 } \Phi\left(c_{(1)}\right) \Phi\left(e_{A}\right) \Phi^{-1}\left(c_{(2)}{ }^{A}{ }_{(1)}\right) \otimes c_{(2)}{ }^{A}{ }_{(2)} \\
& \text { 且 } \Phi\left(c_{(1)}\right) \Phi(e)_{\alpha} \Phi^{-1}\left(c_{(2)}{ }^{\alpha}{ }_{(1)}\right) \otimes c_{(2)}{ }^{\alpha}{ }_{(2)}=\Phi\left(c_{(1)}\right) \Phi^{-1}\left(c_{(2)}\right) \otimes c_{(3)} \\
& =1 \otimes c \text {. }
\end{aligned}
$$

Therefore $\rho$ and $\sigma$ satisfy conditions (i)-(iv). To prove the remaining part of the example we use the observation made in [5] that $M \otimes C$ is isomorphic to $P$ as a vector space with the isomorphism $\Theta: x \otimes c \rightarrow x \Phi(c)$. It suffices to show that $\Theta$ is an algebra isomorphism. We have

$$
\begin{aligned}
\Theta((x \otimes b)(y \otimes c)) & =x \Phi\left(b_{(1)}\right) y_{\alpha} \Phi\left(c_{A}\right) \Phi^{-1}\left(b_{(2)}{ }^{\alpha A}{ }_{(1)}\right) \Phi\left(b_{(2)}^{\alpha A}{ }_{(2)}\right) \\
& \left.=x \Phi\left(b_{(1)}\right) y_{\alpha} \Phi\left(c_{A}\right) \epsilon{\left(b_{(2)}\right.}^{\alpha A}\right) \stackrel{\text { 孟 })}{=} x \Phi(b) y \Phi(c)=\Theta(x \otimes b) \Theta(y \otimes c) .
\end{aligned}
$$


Therefore $M \rtimes_{\Phi} C$ is isomorphic to $P$ as an algebra and since $P$ is associative, so is $M \rtimes_{\Phi} C$.

Since $P$ and $C$ are entwined by $\psi$, and $P$ is isomorphic to $M \rtimes_{\Phi} C$ as an algebra, one would expect that the cleft extension $M \rtimes_{\Phi} C$ and $C$ are entwined by $\tilde{\psi}=\left(\Theta^{-1} \otimes \operatorname{id}_{C}\right) \circ \psi \circ\left(\operatorname{id}_{C} \otimes \Theta\right)$. This is not always the case, however. Instead we have

Lemma 2.6 $M \rtimes_{\Phi} C$ and $C$ are entwined by $\tilde{\psi}$ if and only if $\psi^{C}(c \otimes e)=e \otimes c$, for any $c \in C$.

Proof. Assume first that the hypothesis of the lemma is satisfied. Then first of conditions (更) is satisfied by $\tilde{\psi}$ since $\Theta$ is an algebra map. Explicitly, we have

$$
\begin{aligned}
\tilde{\psi} \circ\left(\operatorname{id}_{C} \otimes \mu\right) & =\left(\Theta^{-1} \otimes \operatorname{id}_{C}\right) \circ \psi \circ\left(\operatorname{id}_{C} \otimes \Theta\right) \circ\left(\operatorname{id}_{C} \otimes \mu\right) \\
& =\left(\Theta^{-1} \otimes \operatorname{id}_{C}\right) \circ \psi \circ\left(\operatorname{id}_{C} \otimes \mu\right) \circ\left(\operatorname{id}_{C} \otimes \Theta \otimes \Theta\right) \\
& =\left(\Theta^{-1} \otimes \mathrm{id}_{C}\right) \circ\left(\mu \otimes \operatorname{id}_{C}\right) \circ \psi_{12} \circ \psi_{23} \circ\left(\operatorname{id}_{C} \otimes \Theta \otimes \Theta\right) \\
& =\left(\mu \otimes \mathrm{id}_{C}\right) \circ\left(\Theta^{-1} \otimes \Theta^{-1} \otimes \operatorname{id}_{C}\right) \circ \psi_{12} \circ \psi_{23} \circ\left(\operatorname{id}_{C} \otimes \Theta \otimes \Theta\right) \\
& =\left(\mu \otimes \mathrm{id}_{C}\right) \circ \tilde{\psi}_{12} \circ \tilde{\psi}_{23} .
\end{aligned}
$$

Here we used $\mu$ to denote the products in $P$ and in $M \rtimes_{\Phi} C$. To prove the second of conditions (回) we first notice, that $\tilde{\psi}$ can be explicitly written as

$$
\tilde{\psi}(b \otimes x \otimes c)=x_{\alpha} \otimes c_{A} \otimes b^{\alpha A}
$$

for any $b, c \in C$ and $x \in M$. In particular

$$
\tilde{\psi}(b \otimes 1 \otimes e)=1_{\alpha} \otimes e_{A} \otimes b^{\alpha A}=1 \otimes e_{A} \otimes b^{A}=1 \otimes e \otimes b
$$

where we used the hypothesis to derive the last equality. The verification of conditions (2) is easy.

Conversely, if we assume that $\tilde{\psi}$ entwines $M \rtimes_{\Phi} C$ with $C$ then the second of conditions (四) will imply

$$
1 \otimes e \otimes c=1_{\alpha} \otimes e_{A} \otimes c^{\alpha A}=1 \otimes e_{A} \otimes c^{A}
$$

and thus the assertion follows.

\section{Equivalent Crossed Products}


Proposition 3.1 Let $M \rtimes_{\rho, \sigma} C$ be a crossed product algebra as in Proposition 2.1 associated to the entwining data $\left(P, C, \psi, e, \psi^{C}\right), M=P_{e}^{c o C}$. Let $\gamma: C \rightarrow M$ be a convolution invertible map such that $\gamma(e)=1$ and

$$
\psi_{23}^{C} \circ \psi_{12} \circ\left(\operatorname{id}_{C} \otimes \gamma \otimes \operatorname{id}_{C}\right) \circ\left(\operatorname{id}_{C} \otimes \Delta\right)=\left(\gamma \otimes \operatorname{id}_{C} \otimes \operatorname{id}_{C}\right) \circ\left(\Delta \otimes \operatorname{id}_{C}\right) \circ \psi^{C} .
$$

Define the maps $\rho^{\prime}: C \otimes P \rightarrow P$ and $\sigma^{\prime}: C \otimes C \rightarrow M$ by

$$
\begin{gathered}
\rho^{\prime}(c, u)=\gamma\left(c_{(1)}\right) \rho\left(c_{(2)}, u_{\alpha}\right) \gamma^{-1}{\left(c_{(3)}{ }^{\alpha}\right)} \\
\sigma^{\prime}(b, c)=\gamma\left(b_{(1)}\right) \rho\left(b_{(2)}, \gamma\left(c_{A(1)}\right)_{\alpha}\right) \sigma\left(b_{(3)}^{\alpha}, c_{A(2)}\right) \gamma^{-1}\left(b_{(4)}{ }^{A}\right) .
\end{gathered}
$$

Then $\left(\rho^{\prime}, \sigma^{\prime}\right)$ are the crossed product data and $M \rtimes_{\rho^{\prime}, \sigma^{\prime}} C$ is isomorphic to $M \rtimes_{\rho, \sigma} C$ as an algebra. We say that the crossed product data $(\rho, \sigma)$ and $\left(\rho^{\prime}, \sigma^{\prime}\right)$ are gauge equivalent.

Proof. Clearly the output of $\sigma^{\prime}$ is in $M$ by condition (ii) for $\rho$. We now check that $\rho^{\prime}$ and $\sigma^{\prime}$ satisfy conditions (i)-(iv) of Proposition 2.1. Property (i) is immediate. Also, using (2) and the property (ii) of $\rho$ we immediately obtain the property (ii) of $\rho^{\prime}$. To verify (iii) we take any $c \in C, x, y \in M$ and compute

$$
\begin{aligned}
& \left.\rho^{\prime}(c, x y)=\gamma\left(c_{(1)}\right) \rho\left(c_{(2)},(x y)_{\alpha}\right) \gamma^{-1}{\left(c_{(3)}\right.}^{\alpha}\right) \stackrel{\text { 这 }}{\underline{\underline{n}}} \gamma\left(c_{(1)}\right) \rho\left(c_{(2)}, x_{\alpha} y_{\beta}\right) \gamma^{-1}\left(c_{(3)}{ }^{\alpha \beta}\right) \\
& =\gamma\left(c_{(1)}\right) \rho\left(c_{(2)}, x_{\alpha \delta}\right) \rho\left(c_{(3)}{ }^{\delta}, y_{\beta}\right) \gamma^{-1}\left(c_{(4)}{ }^{\alpha \beta}\right) \\
& \text { 国 } \gamma\left(c_{(1)}\right) \rho\left(c_{(2)}, x_{\alpha}\right) \rho\left(c_{(3)}{ }^{\alpha}{ }_{(1)}, y_{\beta}\right) \gamma^{-1}\left(c_{(3)}{ }^{\alpha}{ }_{(2)}{ }^{\beta}\right) \\
& =\gamma\left(c_{(1)}\right) \rho\left(c_{(2)}, x_{\alpha}\right) \gamma^{-1}\left(c_{(3)}{ }^{\alpha}{ }_{(1)}\right) \gamma\left(c_{(3)}{ }^{\alpha}{ }_{(2)}\right) \rho\left(c_{(3)}{ }^{\alpha}{ }_{(3)}, y_{\beta}\right) \gamma^{-1}{\left(c_{(3)}{ }^{\alpha}{ }_{(4)}{ }^{\beta}\right)} \\
& \text { 丝 } \gamma\left(c_{(1)}\right) \rho\left(c_{(2)}, x_{\alpha \delta}\right) \gamma^{-1}\left(c_{(3)}{ }^{\delta}\right) \gamma\left(c_{(4)}{ }^{\alpha}{ }_{(1)}\right) \rho\left(c_{(4)}{ }^{\alpha}{ }_{(2)}, y_{\beta}\right) \gamma^{-1}\left(c_{(4)}{ }^{\alpha}{ }_{(3)}{ }^{\beta}\right) \\
& =\rho^{\prime}\left(c_{(1)}, x_{\alpha}\right) \rho^{\prime}\left(c_{(2)}^{\alpha}, y\right) \text {. }
\end{aligned}
$$

In the third equality we used the fact that $\rho$ has the property (iii).

To verify (iv) we take any $c \in C$ and compute

$$
\begin{aligned}
\sigma^{\prime}(e, c) & =\gamma(e) \rho\left(e, \gamma\left(c_{A(1)}\right)_{\alpha}\right) \sigma\left(e^{\alpha}, c_{A(2)}\right) \gamma^{-1}\left(e^{A}\right) \\
& =\rho\left(e, \gamma\left(c_{A(1)}\right)\right) \sigma\left(e, c_{A(2)}\right) \gamma^{-1}\left(e^{A}\right)=\gamma\left(c_{A}\right) \gamma^{-1}\left(e^{A}\right)=\epsilon(c)
\end{aligned}
$$

Furthermore

$$
\begin{aligned}
& \sigma^{\prime}\left(c_{(1)}, e_{A}\right) \otimes c_{(2)}{ }^{A}=\gamma\left(c_{(1)}\right) \rho\left(c_{(2)}, \gamma\left(e_{A B(1)}\right) \alpha\right) \sigma\left(c_{(3)}{ }^{\alpha}, e_{A B(2)}\right) \gamma^{-1}\left(c_{(4)}^{B}\right) \otimes c_{(5)}{ }^{A}
\end{aligned}
$$

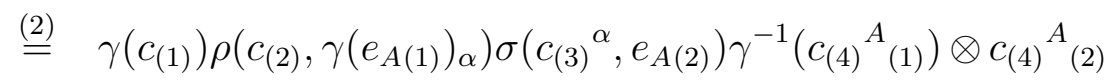

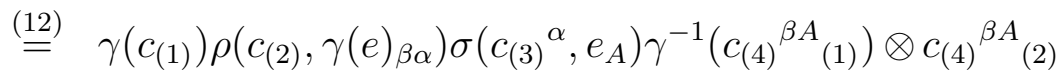

$$
\begin{aligned}
& \text { 国 } \gamma\left(c_{(1)}\right) \rho\left(c_{(2)}, 1\right) \sigma\left(c_{(3)}{ }^{\alpha}, e_{A}\right) \gamma^{-1}{\left(c_{(4)}{ }^{\beta A}{ }_{(1)}\right) \otimes c_{(4)} \beta A_{(2)}} \\
& =\gamma\left(c_{(1)}\right) \gamma^{-1}\left(c_{(2)}\right) \otimes c_{(3)}=1 \otimes c \text {. }
\end{aligned}
$$


Therefore $\rho^{\prime}$ and $\sigma^{\prime}$ satisfy condition (i)-(iv) of Proposition 2.1. Therefore we can define a map $\mu: M \otimes C \otimes M \otimes C \rightarrow M \otimes C$ by (四) with $\rho$ replaced by $\rho^{\prime}$ and $\sigma$ replaced by $\sigma^{\prime}$. We consider a linear map $\Theta: M \otimes C \rightarrow M \rtimes_{\rho, \sigma} C$ given by

$$
\Theta(x \otimes c)=x \gamma\left(c_{(1)}\right) \otimes c_{(2)}
$$

It is clearly an isomorphism of vector spaces. We will show that for all $x, y \in M, b, c \in C$ $\Theta(\mu(x \otimes b \otimes y \otimes c))=\Theta(x \otimes b) \Theta(y \otimes c)$. To do so we first compute the map $\mu$

$$
\begin{aligned}
& \mu(x \otimes b \otimes y \otimes c) \quad=\quad x \gamma\left(b_{(1)}\right) \rho\left(b_{(2)}, y_{\beta \alpha}\right) \gamma^{-1}\left(b_{(3)}{ }^{\beta}\right) \gamma\left(b_{(4)}{ }^{\alpha}{ }_{(1)}\right) \rho\left(b_{(4)}{ }^{\alpha}{ }_{(2)}, \gamma\left(c_{A B(1)}\right)_{\delta}\right) \times \\
& \times \sigma\left(b_{(4)}{ }^{\alpha}{ }_{(3)}{ }^{\delta}, c_{A B(2)}\right) \gamma^{-1}\left(b_{(4)}{ }^{\alpha}{ }_{(4)}{ }^{B}\right) \otimes b_{(4)}{ }^{\alpha}{ }_{(4)}{ }^{A} \\
& \text { 国 } x \gamma\left(b_{(1)}\right) \rho\left(b_{(2)}, y_{\alpha}\right) \rho\left(b_{(3)}{ }^{\alpha}{ }_{(1)}, \gamma\left(c_{A(1)}\right)_{\delta}\right) \sigma\left(b_{(3)}{ }^{\alpha}{ }_{(2)}{ }^{\delta}, c_{A(2)}\right) \gamma^{-1}\left(b_{(3)}{ }^{\alpha}{ }_{(3)}{ }^{A}{ }_{(1)}\right) \\
& \otimes b_{(3)}{ }^{\alpha}{ }_{(3)}^{A}{ }_{(2)} \\
& \underline{\underline{1})} \quad x \gamma\left(b_{(1)}\right) \rho\left(b_{(2)}, y_{\alpha \beta \mu}\right) \rho\left(b_{(3)}^{\mu}, \gamma\left(c_{A(1)}\right)_{\delta}\right) \sigma\left(b_{(4)}^{\beta \delta}, c_{A(2)}\right) \gamma^{-1}\left(b_{(5)}{ }^{\alpha A}{ }_{(1)}\right) \\
& \otimes b_{(5)}^{\alpha A}{ }_{(2)} \\
& =\quad x \gamma\left(b_{(1)}\right) \rho\left(b_{(2)}, y_{\alpha \beta} \gamma\left(c_{A(1)}\right)_{\delta}\right) \sigma\left(b_{(3)}^{\beta \delta}, c_{A(2)}\right) \gamma^{-1}\left(b_{(4)}^{\alpha A_{(1)}}\right) \otimes b_{(4)}^{\alpha A_{(2)}} \\
& \text { 国) } x \gamma\left(b_{(1)}\right) \rho\left(b_{(2)},\left(y_{\alpha} \gamma\left(c_{A(1)}\right)\right)_{\beta}\right) \sigma\left(b_{(3)}^{\beta}, c_{A(2)}\right) \gamma^{-1}\left(b_{(4)}^{\alpha A_{(1)}}\right) \otimes b_{(4)}^{\alpha A_{(2)}} \\
& \text { 12) } x \gamma\left(b_{(1)}\right) \rho\left(b_{(2)},\left(y_{\alpha} \gamma\left(c_{(1)}\right)_{\delta}\right)_{\beta}\right) \sigma\left(b_{(3)}{ }^{\beta}, c_{(2)}\right) \gamma^{-1}{\left(b_{(4)}\right.}^{\left.\alpha \delta A_{(1)}\right) \otimes b_{(4)} \alpha \delta A_{(2)}} \\
& \text { 里. } x \gamma\left(b_{(1)}\right) \rho\left(b_{(2)},\left(y \gamma\left(c_{(1)}\right)\right)_{\alpha}\right) \sigma\left(b_{(3)}{ }^{\alpha}{ }_{(1)}, c_{(2)}\right) \gamma^{-1}\left(b_{(3)}{ }^{\alpha}{ }_{(2)}{ }^{A}{ }_{(1)}\right) \\
& \otimes b_{(3)}{ }^{\alpha}{ }_{(2)}^{A}{ }_{(2)} \text {. }
\end{aligned}
$$

To derive the fourth equality we used the property (iii) of $\rho$. Thus

$$
\begin{aligned}
\Theta(\mu(x \otimes b \otimes y \otimes c)) & =x \gamma\left(b_{(1)}\right) \rho\left(b_{(2)},\left(y \gamma\left(c_{(1)}\right)\right)_{\alpha}\right) \sigma\left(b_{(3)}{ }_{(1)}, c_{(2)} A\right) \otimes b_{(3)}{ }^{\alpha}{ }_{(2)}{ }^{A} \\
& =\Theta(x \otimes b) \Theta(y \otimes c) .
\end{aligned}
$$

Therefore $M \otimes C$ is a unital associative algebra with product $\mu$ and unit $1 \otimes e$. This algebra is a crossed product $M \rtimes_{\rho^{\prime}, \sigma^{\prime}} C$ and it is isomorphic to $M \rtimes_{\rho, \sigma} C$ with isomorphism $\Theta$.

Convolution invertible maps $\gamma: C \rightarrow M$ satisfying (12) were considered in [5] in the context of trivial coalgebra $\psi$-principal bundles. They were termed gauge transformations, since they induce a change of trivialisation in a trivial $\psi$-principal bundle or cleft extension of Example 2.5. Hence the terminology for the crossed product data. It was also shown in [5] that the set of all gauge transformations forms a group with respect to convolution product.

From another point of view Proposition 3.1 generalises the notion of equivalence of bialgebra crossed products of [6] to the case of coalgebra crossed products. Precisely, we say that the crossed products $M \rtimes_{\rho, \sigma} C$ and $M \rtimes_{\rho^{\prime}, \sigma^{\prime}} C$, corresponding to the same entwining data 
$\left(P, C, \psi, e, \psi^{C}\right)$ are equivalent if there exists an algebra and a left $M$-module isomorphism $\Theta: M \rtimes_{\rho^{\prime}, \sigma^{\prime}} C \rightarrow M \rtimes_{\rho, \sigma} C$, such that

$$
\psi_{23}^{C} \circ \psi_{12} \circ\left(\operatorname{id}_{C} \otimes \tilde{\Theta}\right)=\left(\tilde{\Theta} \otimes \operatorname{id}_{C}\right) \circ \psi^{C},
$$

where $\tilde{\Theta}(c)=\Theta(1 \otimes c)$. Notice, that in particular (16) implies that $\Theta$ is a right $C$-comodule isomorphism, i.e. $\Delta_{R} \circ \Theta=\left(\Theta \otimes \mathrm{id}_{C}\right) \circ \Delta$, where $\Delta_{R}$ is as in Lemma 2.4.

Proposition 3.2 Crossed products $M \rtimes_{\rho, \sigma} C$ and $M \rtimes_{\rho^{\prime}, \sigma^{\prime}} C$, corresponding to the same entwining data $\left(P, C, \psi, e, \psi^{C}\right)$ are equivalent if and only if the crossed product data $(\rho, \sigma)$ and $\left(\rho^{\prime}, \sigma^{\prime}\right)$ are gauge equivalent.

Proof. If $(\rho, \sigma)$ and $\left(\rho^{\prime}, \sigma^{\prime}\right)$ are gauge equivalent then the map $\Theta$ (15) is an algebra and a left $M$-module isomorphism. Furthermore $\tilde{\Theta}(c)=\gamma\left(c_{(1)}\right) \otimes c_{(2)}=\left(\gamma \otimes \mathrm{id}_{C}\right) \circ \Delta$. Using (12) we then immediately obtain (16).

Conversely, if $\Theta: M \rtimes_{\rho^{\prime}, \sigma^{\prime}} C \rightarrow M \rtimes_{\rho, \sigma} C$ is an algebra and a left $M$-module isomorphism such that (16) holds then we define the map $\gamma: C \rightarrow M$ by $\gamma=\left(\operatorname{id}_{M} \otimes \epsilon\right) \circ \tilde{\Theta}$. Using (16) and the properties of the coaction $\Delta_{R}$ we obtain

$$
\begin{aligned}
L H S & =\psi_{23}^{C} \circ \psi_{12} \circ\left(\mathrm{id}_{C} \otimes \gamma \otimes \mathrm{id}_{C}\right) \circ(\mathrm{id} \otimes \Delta) \\
& =\psi_{23}^{C} \circ \psi_{12} \circ\left(\mathrm{id}_{C} \otimes \mathrm{id}_{M} \otimes \epsilon \otimes \mathrm{id}_{C}\right) \circ\left(\mathrm{id}_{C} \otimes \tilde{\Theta} \otimes \mathrm{id}_{C}\right) \circ\left(\mathrm{id}_{C} \otimes \Delta\right) \\
& =\psi_{23}^{C} \circ \psi_{12} \circ\left(\mathrm{id}_{C} \otimes \mathrm{id}_{M} \otimes \epsilon \otimes \mathrm{id}_{C}\right) \circ\left(\operatorname{id}_{C} \otimes \Delta_{R}\right) \circ\left(\mathrm{id}_{C} \otimes \tilde{\Theta}\right) \\
& =\psi_{23}^{C} \circ \psi_{12} \circ\left(\mathrm{id}_{C} \otimes \tilde{\Theta}\right)=\left(\tilde{\Theta} \otimes \mathrm{id}_{C}\right) \circ \psi^{C} .
\end{aligned}
$$

On the other hand

$$
\begin{aligned}
\text { RHS } & =\left(\gamma \otimes \mathrm{id}_{C} \otimes \mathrm{id}_{C}\right) \circ\left(\Delta \otimes \mathrm{id}_{C}\right) \circ \psi^{C} \\
& =\left(\operatorname{id}_{M} \otimes \epsilon \otimes \mathrm{id}_{C} \otimes \mathrm{id}_{C}\right) \circ\left(\tilde{\Theta} \otimes \mathrm{id}_{C} \otimes \mathrm{id}_{C}\right) \circ\left(\Delta \otimes \mathrm{id}_{C}\right) \circ \psi^{C} \\
& =\left(\operatorname{id}_{M} \otimes \epsilon \otimes \mathrm{id}_{C} \otimes \mathrm{id}_{C}\right) \circ\left(\Delta_{R} \otimes \mathrm{id}_{C}\right) \circ\left(\tilde{\Theta} \otimes \mathrm{id}_{C}\right) \circ \psi^{C}=\left(\tilde{\Theta} \otimes \operatorname{id}_{C}\right) \circ \psi^{C} .
\end{aligned}
$$

Therefore (12) holds. Clearly, $\gamma$ is convolution invertible and $\gamma(e)=1$. Moreover $\Theta(x \otimes c)=$ $x \gamma\left(c_{(1)}\right) \otimes c_{(2)}$. Since $\Theta$ is an algebra map the following expressions

$$
\begin{aligned}
& \Theta(1 \otimes b) \Theta(x \otimes c)=\gamma\left(b_{(1)}\right) \rho\left(b_{(2)}, x_{\alpha} \gamma\left(c_{(1)}\right)_{\beta}\right) \sigma\left(b_{(3)}{ }^{\alpha \beta}{ }_{(1)}, c_{(2)} A\right) \otimes b_{(3)}{ }^{\alpha \beta}{ }_{(2)}{ }^{A}, \\
& \Theta((1 \otimes b)(x \otimes c))=x \rho^{\prime}\left(b_{(1)}, x_{\alpha}\right) \sigma^{\prime}\left(b_{(2)}{ }^{\alpha}{ }_{(1)}, c_{A}\right) \gamma\left(b_{(2)}{ }^{\alpha}{ }_{(2)}{ }_{(1)}^{A}\right) \otimes b_{(2)}{ }^{\alpha}{ }_{(2)}{ }^{A}{ }_{(2)}
\end{aligned}
$$

are equal to each other. Applying $\operatorname{id}_{M} \otimes \gamma^{-1}$ we thus deduce that

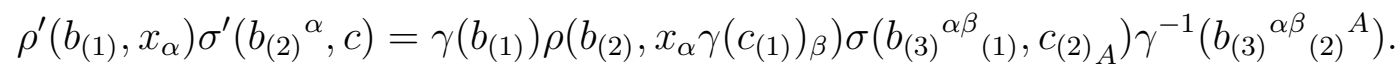


Setting $c=e$ in (17) and using property (iv) of $\sigma$ and $\sigma^{\prime}$ we obtain (13), and setting $x=1$ in (17) we obtain (14). Therefore $(\rho, \sigma)$ and $\left(\rho^{\prime}, \sigma^{\prime}\right)$ are gauge equivalent.

Because any $\sigma$ satisfying condition (5) may be viewed as a generalisation of a cocycle, the results of Proposition 3.1 and Proposition 3.2 lead to the non Abelian cohomology theory generalising that of [11, Section 6.3]. To define a coboundary, however, one would need to assume the existence of a trivial cocycle $\sigma_{\text {triv }}(b, c)=\epsilon(b) \epsilon(c)$. This imposes additional conditions on the entwining data.

Lemma 3.3 The sufficient and necessary conditions for the existence of the trivial cocycle $\sigma_{\text {triv }}$ in the entwining data $\left(P, C, \psi, e, \psi^{C}\right)$, with any map $\rho$ satisfying (i)-(iii) in Proposition 2.1 are

$$
\epsilon\left(e_{A}\right) c^{A}=c, \quad \epsilon\left(c_{A}\right) \epsilon\left(b^{A}{ }_{B}\right) a^{B}=\epsilon\left(b_{A}\right) \epsilon\left(c_{B}\right) a^{A B},
$$

for any $a, b, c \in C$

Proof. In an elementary way one finds that first of conditions (18) ensures second of the properties (iv) of $\sigma_{\text {triv }}$, while the second part of (18) is needed for the cocycle property (5) of $\sigma_{\text {triv }}$.

If the conditions of Lemma 3.3 are satisfied one defines a coboundary as a cocycle equivalent to the trivial one, i.e.

$$
\sigma(b, c)=\gamma\left(b_{(1)}\right) \rho\left(b_{(2)}, \gamma\left(c_{A}\right)\right) \gamma^{-1}\left(b_{(3)}{ }^{A}\right) .
$$

For example, conditions of Lemma 3.3 are satisfied for braided bialgebra crossed products of Example 2.3, therefore the coboundary can be defined and the corresponding cohomology theory developed in this case.

\section{$4 E_{q}(2)$ as a Crossed Product}

In this section we give an explicit example of a crossed product algebra as defined in Proposition 2.1, namely, we show that the two-dimensional quantum Euclidean group $E_{q}(2)$ is a cleft extension as in Example 2.5, $E_{q}(2)=X_{q} \rtimes_{\Phi} C$, where $X_{q}$ is the quantum hyperboloid 13] and $C$ is spanned by group-like elements $c_{p}, p \in \mathbf{Z}$. The algebra $P=E_{q}(2)$ is generated by the elements $v, v^{-1}, n, \bar{n}$ subject to the relations [16] [17]

$$
v n=q^{2} n v, \quad v \bar{n}=q^{2} \bar{n} v, \quad n \bar{n}=q^{2} \bar{n} n \quad v v^{-1}=v^{-1} v=1,
$$


where $q \in k^{*}$. One can define a Hopf algebra structure on $E_{q}(2)$, but this is not important in our construction. Instead we define the entwining structure $\psi: C \otimes P \rightarrow P \otimes C$ by

$$
\begin{aligned}
& \psi\left(c_{p} \otimes v\right)=v \otimes c_{p+1}, \quad \psi\left(c_{p} \otimes n\right)=n \otimes c_{p}+\mu q^{2 p} v \otimes c_{p}-\mu q^{2 p} v \otimes c_{p+1}, \\
& \psi\left(c_{p} \otimes v^{-1}\right)=v^{-1} \otimes c_{p-1}, \quad \psi\left(c_{p} \otimes \bar{n}\right)=\bar{n} \otimes c_{p}+\nu q^{2 p} v^{-1} \otimes c_{p}-\nu q^{2 p} v^{-1} \otimes c_{p-1},
\end{aligned}
$$

where $\mu, \nu \in k^{*}$ or $\mu=\nu=0$. One extends $\psi$ to the whole of $P$ requiring that (国) be satisfied. To prove that such an extension is well-defined one needs to show that

$$
\begin{gathered}
\psi\left(c_{p} \otimes\left(v n-q^{2} n v\right)\right)=\psi\left(c_{p} \otimes\left(v \bar{n}-q^{2} \bar{n} v\right)\right)=0 \\
\psi\left(c_{p} \otimes\left(n \bar{n}-q^{2} \bar{n} n\right)\right)=\psi\left(c_{p} \otimes\left(v v^{-1}-1\right)\right)=\psi\left(c_{p} \otimes\left(v^{-1} v-1\right)\right)=0 .
\end{gathered}
$$

This can be done by elementary computations. It is also clear that (2) holds. Therefore $\psi$ defines the entwining structure for $P$ and $C$. Next we choose $e=c_{s}$ and define the right coaction of $C$ on $P$ by $\Delta_{R}(u)=\psi\left(c_{s} \otimes u\right)$. When $\mu=\nu=0, P$ becomes a right $C$-comodule algebra provided $C$ is equipped with the algebra structure of $k\left[c, c^{-1}\right]$ by $c^{p}=c_{p+s}$, and therefore we do not discuss this case any further. Following [3] one easily finds that the fixed point subalgebra $M$ of $P$ is generated by $z=v+\mu^{-1} q^{-2 s} n$ and $\bar{z}=v^{-1}+\nu^{-1} q^{-2 s} \bar{n}$. The generators $z, \bar{z}$ satisfy the relation $z \bar{z}=q^{2} \bar{z} z+\left(1-q^{2}\right)$, and therefore $M$ is isomorphic to the quantum hyperboloid $X_{q}$.

To complete the entwining data we define $\psi^{C}: C \otimes C \rightarrow C \otimes C$ by $\psi^{C}\left(c_{p} \otimes c_{r}\right)=$ $c_{r} \otimes c_{p+r-s}$. It celarly satisfies conditions (3).

Proposition 4.1 Let $\Phi: C \rightarrow E_{q}(2)$ be a linear map given by $\Phi\left(c_{p}\right)=v^{p-s}$. Then $\Phi$ is convolution invertible and it satisfies (8). Therefore $E_{q}(2)=X_{q} \rtimes_{\Phi} C$ with a trivial cocycle $\sigma\left(c_{p}, c_{r}\right)=1$ and the map $\rho: C \otimes E_{q}(2) \rightarrow E_{q}(2)$,

$$
\rho\left(c_{p}, v^{ \pm 1}\right)=1, \quad \rho\left(c_{p}, n\right)=q^{2 p}\left(q^{-2 s} n+\mu(v-1)\right), \quad \rho\left(c_{p}, \bar{n}\right)=q^{2 p}\left(q^{-2 s} \bar{n}+\nu\left(v^{-1}-1\right)\right) .
$$

Proof. Clearly $\Phi$ is convolution invertible and $\Phi^{-1}\left(c_{p}\right)=v^{-p+s}$. Also, $\Phi(e)=\Phi\left(c_{s}\right)=1$. To check that $\Phi$ satisfies (8) we compute

$$
\psi\left(c_{p} \otimes \Phi\left(c_{r}\right)\right)=\psi\left(c_{p} \otimes v^{r-s}\right)=v^{r-s} \otimes c_{p+r-s}=\Phi\left(c_{r}\right) \otimes c_{p+r-s}=\left(\Phi \otimes \mathrm{id}_{C}\right) \circ \psi^{C}\left(c_{p} \otimes c_{r}\right) .
$$

By Example 2.5 $E_{q}(2)=X_{q} \rtimes_{\rho, \sigma} C$, with $\rho$ and $\sigma$ as specified.

Although one can easily define a Hopf algebra structure on $C$ (e.g. $C$ could be a group algebra of any group $G$ such that $\# G=\# \mathbf{Z})$ one equally easily finds that $E_{q}(2)$ is never a right $C$-comodule algebra except when $(\mu, \nu)=(0,0)$. Furthermore, because $\psi\left(C \otimes X_{q}\right)$ 
is not a subset of $X_{q} \otimes C$ there is no braiding making $E_{q}(2)$ a braided $C$-comodule algebra. Therefore the notion of a coalgebra crossed product developed in this paper is truly needed for description of $E_{q}(2)$.

Acknowledgements. The studies of crossed products by a coalgebra were triggered by the work on coalgebra gauge theory [5], a research carried out in collaboration with Shahn Majid. I am grateful to him for continuing discussions.

\section{A Dual Crossed Products}

In [5] it was observed that the definition of the entwining structure $P, C, \psi$ (eqs. (1 12 )) possess the following self-duality property. If one interchanges $P$ with $C, \mu$ with $\Delta$, the unit map $\lambda \mapsto \lambda .1$ with $\epsilon$ and reverses the order of composition of maps then the set of conditions (112) remains unchanged. One can use this self-duality property to dualise the notion of a crossed product algebra and thus obtain the dual crossed product coalgebra. In this section we present the result of such a dualisation.

We begin with an algebra $P$ and a coalgebra $C$ entwined by $\psi$. Assume that there is an algebra character $\kappa: P \rightarrow k$. As shown in [5], the map $(\kappa \otimes \mathrm{id}) \circ \psi$ is a right action of $P$ on $C$. Moreover the linear space $J_{\kappa}=\operatorname{span}\left\{c^{\alpha} \kappa\left(u_{\alpha}\right)-c \kappa(u) \mid c \in C, u \in P\right\}$ is a coideal. Hence $M=C / J_{\kappa}$ is a coalgebra. Finally we assume that there is a linear map $\psi^{P}: P \otimes P \rightarrow P \otimes P$ such that for any $u \in P$

$$
\psi^{P} \circ(\mathrm{id} \otimes \mu)=(\mu \otimes \mathrm{id}) \circ \psi_{23}^{P} \circ \psi_{12}^{P}, \quad \psi^{P}(u \otimes 1)=1 \otimes u, \quad(\kappa \otimes \mathrm{id}) \circ \psi^{P}=\mu .
$$

Notice that (19) comes from condition (3) by the dualisation procedure explained above with the map $k \rightarrow C, \lambda \mapsto \lambda e$ replaced by $\kappa$. We denote $\psi^{P}(u \otimes v)=v_{A} \otimes u^{A}$ (summation understood). We say that $\left(C, P, \psi, \kappa, \psi^{P}\right)$ are dual entwining data. Dualising Proposition 2.1 we thus obtain

Proposition A.1 Let $\left(C, P, \psi, \kappa, \psi^{P}\right)$ be dual entwining data, $M=C / J_{\kappa}$ with a canonical surjection $\pi: C \rightarrow M$, and let $\bar{\rho}: C \rightarrow P \otimes C$ and $\bar{\sigma}: M \rightarrow P \otimes P$ be linear maps. We will denote the action of these maps by $\bar{\rho}(c)=c^{<1>} \otimes c^{<2>}$ and $\bar{\sigma}(m)=m^{(1)} \otimes m^{(2)}$ (summation understood). Assume that for all $j \in J_{\kappa}, m \in M, u \in P$ and $c \in C$ we have:

(i') $\kappa\left(c^{<1>}\right) \pi\left(c^{<2>}\right)=\pi(c), \quad c^{<1>} \epsilon\left(c^{<2>}\right)=\epsilon(c)$;

(ii') $j^{<1>} u_{\alpha} \otimes j^{<2>^{\alpha}} \in P \otimes J_{\kappa}$;

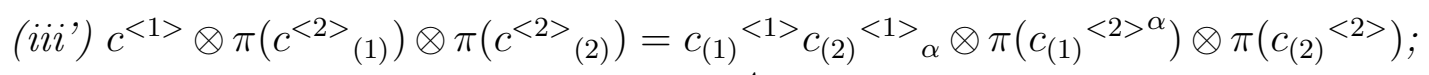

$\left(i v^{\prime}\right) \kappa\left(m^{(1)}\right) m^{(2)}=\epsilon(m), \quad m^{(1)} u_{A} \kappa\left(m^{(2)^{A}}\right)=\epsilon(m) u$. 
Then the vector space $M \otimes P$ is a coalgebra with a coproduct

$$
\Delta(m \otimes u)=\pi\left(c_{(1)}\right) \otimes c_{(2)}^{<1>}\left(\pi\left({ }_{(3)}\right)^{(1)} u_{A}\right)_{\alpha} \otimes \pi\left(c_{(2)}^{<2>^{\alpha}}\right) \otimes \pi\left(c_{(3)}\right)^{(2)^{A}}
$$

where $c \in \pi^{-1}(m)$, and a counit $\epsilon \otimes \kappa$ if and only if for any $u \in P$ and $c \in C, m=\pi(c)$

$$
\begin{aligned}
& c_{(1)}^{<1>}\left(\pi\left(c_{(2)}\right)^{(1)} u_{A}\right)_{\alpha} \otimes \pi\left(c_{(1)}^{<2>\alpha}\right)^{(1)} \pi\left(c_{(2)}\right)^{<2>A}{ }_{B} \otimes \pi\left(c_{(1)}^{<2>\alpha}\right)^{(2)^{B}} \\
& =m_{(1)}{ }^{(1)}\left(m_{(2)}{ }^{(1)} u_{A}\right)_{B} \otimes m_{(1)}^{(2)}{ }^{B} \otimes m_{(2)}{ }^{(2)^{A}} \text {, }
\end{aligned}
$$

and

$$
\begin{aligned}
& c_{(1)}^{<1>}\left(\pi\left(c_{(2)}\right)^{(1)} u_{A}\right)_{\alpha} \otimes c_{(1)}{ }^{<2>} \alpha_{<1>} \pi\left(c_{(2)}\right)^{(2)^{A}}{ }_{\beta} \otimes \pi\left(c_{(1)}{ }^{<2>} \alpha_{<2>\beta}\right) \\
& =\pi\left(c_{(1)}\right)^{(1)}\left(c_{(2)}^{<1>} u_{\alpha}\right)_{A} \otimes \pi\left(c_{(1)}\right)^{(2)^{A} \otimes c_{(2)}<2>\alpha} \text {. }
\end{aligned}
$$

We denote the resulting coalgebra by $M \rtimes^{\bar{\rho}, \bar{\sigma}} P$ and call it a dual crossed product coalgebra. We call $(\bar{\rho}, \bar{\sigma})$ the dual crossed product data

The proof of Proposition A.1 is a straightforward dualisation of the proof of Proposition 2.1 and thus we do not write it here. We only remark that the map $\Delta(20)$ is welldefined by condition (ii') and that the surjection $\pi$ dualises the natural inclusion $M \hookrightarrow P$ consequently omitted in Sections 2 and 3.

We now give an example of a dual crossed product coalgebra which is obtained by the dualisation of Example 2.5.

Example A.2 Let $\left(C, P, \psi, \kappa, \psi^{P}\right)$ be dual entwining data, $M=C / J_{\kappa}$ with a canonical surjection $\pi: C \rightarrow M$, and let $\Phi: C \rightarrow P$ be a convolution invertible map such that $\kappa \circ \Phi=\epsilon$ and

$$
\left(\mathrm{id}_{P} \otimes \Phi\right) \circ \psi=\psi^{P} \circ\left(\Phi \otimes \mathrm{id}_{P}\right) .
$$

Define the maps $\bar{\rho}: C \rightarrow P \otimes C$ and $\bar{\sigma}: M \rightarrow P \otimes P$ by

$$
\bar{\rho}(c)=\Phi\left(c_{(1)}\right) \Phi^{-1}\left(c_{(3)}\right)_{\alpha} \otimes c_{(2)}{ }^{\alpha}, \quad \bar{\sigma}(m)=\Phi\left(b_{(1)}\right) \Phi^{-1}\left(b_{(3)}\right)_{A} \otimes \Phi\left(b_{(2)}\right)^{A}
$$

where $b \in \pi^{-1}(m)$. Then there is a dual crossed product coalgebra $M \rtimes^{\bar{\rho}, \bar{\sigma}} P$. The coproduct in $M \rtimes^{\bar{\rho}, \bar{\sigma}} P$ reads explicitly

$$
\Delta(m \otimes u)=\pi\left(c_{(1)}\right) \otimes \Phi\left(c_{(2)}\right)\left(\Phi^{-1}\left(c_{(5)}\right) u\right)_{A \alpha} \otimes \pi\left(c_{(3)}{ }^{\alpha}\right) \otimes \Phi\left(c_{(4)}\right)^{A},
$$

where $c \in \pi^{-1}(m)$, and $M \rtimes^{\bar{\rho}, \bar{\sigma}} P \cong C$ as coalgebras. 
Proof. This example is a dualisation of Example 2.5 and hence it can be proved following the same steps as in the proof of Example 2.5. Therefore we do not repeat the full proof in here. We only show that the map $\bar{\sigma}$ is well defined and we write the explicit form of a coalgebra isomorphism $M \rtimes^{\bar{\rho}, \bar{\sigma}} P \cong C$. To prove the former we denote the function on the right hand side of definition (25) of $\bar{\sigma}$ by $\tilde{\sigma}$ and compute

$$
\begin{aligned}
& \tilde{\sigma}\left(\kappa\left(u_{\alpha}\right) c^{\alpha}\right) \stackrel{2}{2} \quad \kappa\left(u_{\alpha \beta \gamma}\right) \Phi\left(c_{(1)}^{\gamma}\right) \Phi^{-1}\left(c_{(3)}{ }^{\alpha}\right)_{A} \otimes \Phi\left(c_{(2)}{ }^{\beta}\right)^{A} \\
& \text { 23) } \kappa\left(u_{\alpha \beta B}\right) \Phi\left(c_{(1)}\right)^{B} \Phi^{-1}\left(c_{(3)}{ }^{\alpha}\right)_{A} \otimes \Phi\left(c_{(2)}{ }^{\beta}\right)^{A} \\
& \text { 19) } \Phi\left(c_{(1)}\right) u_{\alpha \beta} \Phi^{-1}\left(c_{(3)}{ }^{\alpha}\right)_{A} \otimes \Phi\left(c_{(2)}^{\beta}\right)^{A} \\
& \text { 23] } \Phi\left(c_{(1)}\right) u_{\alpha B} \Phi^{-1}\left(c_{(3)}^{\alpha}\right)_{A} \otimes \Phi\left(c_{(2)}\right)^{B A} \\
& \text { 19) } \Phi\left(c_{(1)}\right)\left(u_{\alpha} \Phi^{-1}\left(c_{(3)}{ }^{\alpha}\right)\right)_{A} \otimes \Phi\left(c_{(2)}\right)^{A}=\kappa(u) \Phi\left(c_{(1)}\right) \Phi^{-1}\left(c_{(3)}\right) \otimes \Phi\left(c_{(2)}\right) \\
& =\tilde{\sigma}(\kappa(u) c) \text {. }
\end{aligned}
$$

To derive the penultimate equality we used the following property of $\Phi^{-1}$,

$$
\Phi^{-1}(c) \kappa(u)=u_{\alpha} \Phi^{-1}\left(c^{\alpha}\right),
$$

which can be easily derived from (23) or alternatively obtained from (11) by dualisation. Therefore $J_{\kappa} \subset \operatorname{ker} \tilde{\sigma}$ and the map $\bar{\sigma}$ is well-defined.

The coalgebra isomorphism $\Theta: C \rightarrow M \rtimes^{\bar{\rho}, \bar{\sigma}} P$ and its inverse are given by

$$
\Theta(c)=\pi\left(c_{(1)}\right) \otimes \Phi\left(c_{(2)}\right), \quad \Theta^{-1}(\pi(c) \otimes u)=\kappa\left(\left(\Phi^{-1}\left(c_{(2)}\right) u\right)_{\alpha}\right) c_{(1)}{ }^{\alpha}
$$

To see that the map $\Theta^{-1}$ is well-defined we denote the right hand side of definition (27) of $\Theta^{-1}$ by $\theta$ and compute

$$
\begin{array}{cl}
\theta\left(\kappa\left(v_{\gamma}\right) c^{\gamma} \otimes u\right) & \stackrel{\text { (日) }}{ } \kappa\left(v_{\gamma \beta}\left(\Phi^{-1}\left(c_{(2)}^{\gamma}\right) u\right)_{\alpha}\right) c_{(1)} \beta \alpha \underline{\underline{1})} \kappa\left(\left(v_{\beta} \Phi^{-1}\left(c_{(2)}^{\beta}\right) u\right)_{\alpha}\right) c_{(1)}{ }^{\alpha} \\
\text { (2) } & \kappa(v) \kappa\left(\left(\Phi^{-1}\left(c_{(2)}\right) u\right)_{\alpha}\right) c_{(1)}{ }^{\alpha}=\theta(\kappa(v) c \otimes u) .
\end{array}
$$

Therefore $J_{\kappa} \subset \operatorname{ker} \theta$ and $\Theta^{-1}$ is well-defined as stated. The proof that $\Theta$ is a comodule map and that $\Theta^{-1}$ is its inverse can be obtained by careful dualisation of the corresponding parts of the proof of Example 2.5 and hence we do not write it here.

A dual crossed product $M \rtimes^{\bar{\rho}, \bar{\sigma}} P$ is a left $M$-comodule with the coaction $\Delta_{L}(m \otimes u)=$ $m_{(1)} \otimes m_{(2)} \otimes u$. Moreover $M \rtimes^{\bar{\rho}, \bar{\sigma}} P$ is a right $P$-module with the action $m \otimes u \otimes v \mapsto m \otimes u v$. We say that dual crossed products $M \rtimes^{\bar{\rho}, \bar{\sigma}} P$ and $M \rtimes^{\bar{\rho}^{\prime}, \bar{\sigma}^{\prime}} P$ corresponding to dual entwining data $\left(C, P, \psi, \kappa, \psi^{P}\right)$ are equivalent if there exists a coalgebra and a left $M$-comodule isomorphism $\Theta: M \rtimes^{\bar{\rho}, \bar{\sigma}} P \rightarrow M \rtimes^{\bar{\rho}^{\prime}, \bar{\sigma}^{\prime}} P$ such that

$$
\psi^{P} \circ\left(\tilde{\Theta} \otimes \operatorname{id}_{P}\right)=\left(\operatorname{id}_{P} \otimes \tilde{\Theta}\right) \circ \psi_{12} \circ \psi_{23}^{P},
$$


where $\tilde{\Theta}=\left(\epsilon \otimes \mathrm{id}_{P}\right) \circ \Theta$. By dualising Propositions 3.1 and 3.2 we obtain the following

Proposition A.3 Let $\left(C, P, \psi, \kappa, \psi^{P}\right)$ be dual entwining data, $M=C / J_{\kappa}$ with a canonical surjection $\pi: C \rightarrow M$, and let $(\bar{\rho}, \bar{\sigma}),\left(\bar{\rho}^{\prime}, \bar{\sigma}^{\prime}\right)$ be dual crossed product data. Then the following statements are equivalent:

(1) Crossed products $M \rtimes^{\bar{\rho}, \bar{\sigma}} P$ and $M \rtimes^{\bar{\rho}^{\prime}, \bar{\sigma}^{\prime}} P$ are equivalent.

(2) There exists a convolution invertible map $\gamma: M \rightarrow P$ such that $\kappa \circ \gamma=\epsilon$,

$$
\psi^{P} \circ\left(\mu \otimes \mathrm{id}_{P}\right) \circ\left(\gamma \otimes \mathrm{id}_{P} \otimes \mathrm{id}_{P}\right)=\left(\operatorname{id}_{P} \otimes \mu\right) \circ\left(\mathrm{id}_{P} \otimes \gamma \otimes \mathrm{id}_{P}\right) \circ \psi_{12} \circ \psi_{23}^{P},
$$

and such that

$$
\bar{\rho}^{\prime}(c)=\gamma\left(\pi\left(c_{(1)}\right)\right) c_{(2)}^{<1>} \gamma^{-1}\left(\pi\left(c_{(3)}\right)\right)_{\alpha} \otimes c_{(2)}<2>\alpha
$$

and

$$
\bar{\sigma}^{\prime}(\pi(c))=\gamma\left(\pi\left(c_{(1)}\right)\right) c_{(2)}{ }^{<1>} \pi\left(c_{(3)}\right)^{(1)}{ }_{\alpha} \gamma^{-1}\left(\pi\left(c_{(4)}\right)\right)_{A} \otimes\left(\gamma\left(\pi\left(c_{(2)}{ }^{<2>\alpha}\right)\right) \pi\left(c_{(3)}\right)^{(2)}\right)^{A}
$$

where $\bar{\rho}(c)=c^{<1>} \otimes c^{<2>}$ and $\bar{\rho}(m)=m^{(1)} \otimes m^{(2)}$.

As before also this proposition can be proved by following the steps of proofs of Propositions 3.1 and 3.2, therefore we skip the proof again. We only remark that the map $\bar{\sigma}^{\prime}$ is well defined by condition (ii').

In this way we were able to extend all the results of Sections 2 and 3 to the case of dual crossed products.

\section{References}

[1] R.J. Blattner, M. Cohen and S. Montgomery. Crossed products and inner actions of Hopf algebras. Trans. A.M.S., 298:671-711, 1986.

[2] R.J. Blattner and S. Montgomery. Crossed products and Galois extensions of Hopf algebras. Pacific J. Math.., 137:37-54, 1986.

[3] F. Bonechi, N. Ciccoli, R. Giachetti, E. Sorace and M. Tarlini. Free q-Schrödinger equation from homogeneous spaces of the 2-dim Euclidean quantum group. Commun. Math. Phys., 175:161-176, 1996.

[4] T. Brzeziński and S. Majid. Quantum group gauge theory on quantum spaces. Commun. Math. Phys., 157:591-638, 1993. Erratum 167:235, 1995. 
[5] T. Brzeziński and S. Majid. Coalgebra gauge theory. Preprint DAMTP/95-74, @ $\operatorname{alg} / 9602022$.

[6] Y. Doi. Equivalent crossed products for a Hopf algebra. Commun. Alg., 17:3053-3085, 1989.

[7] Y. Doi and M. Takeuchi. Cleft comodule algebras for a bialgebra. Commun. Alg., 14:801-817, 1986.

[8] S. Majid. Braided groups and algebraic quantum field theories. Lett. Math. Phys., 22:167-176, 1991.

[9] S. Majid. Beyond supersymmetry and quantum symmetry (an introduction to braided groups and braided matrices). In M-L. Ge and H.J. de Vega, editors, Quantum Groups, Integrable Statistical Models and Knot Theory, pages 231-282. World Sci., 1993.

[10] S. Majid. Cross products by braided groups and bosonisation. J. Algebra, 163:165-190, 1994.

[11] S. Majid. Foundations of Quantum Group Theory. Cambridge University Press, 1995.

[12] M. Pflaum. Quantum Groups on Fibre Bundles. Commun. Math. Phys. 166:279-315, 1994.

[13] K. Schmüdgen. Integrable Operator Representations of $\mathbf{R}_{q}^{2}, X_{q, \gamma}$ and $S L_{q}(2, \mathbf{R})$. Commun. Math. Phys., 159:217-238, 1994.

[14] H.-J. Schneider. Hopf-Galois Extensions, Crossed Products, and Clifford Theory. In: J. Bergen and S. Montgomery, editors, Advances in Hopf Algebras, pages 267-297, Marcel Dekker, Inc., 1994.

[15] M. Sweedler. Cohomology of algebras over Hopf algebras. Trans. AMS, 133:205-239, 1968.

[16] L.L. Vaksman and L.I. Korogodskii. An algebra of bounded functions on the quantum group of the motions of the plane, and q-analogues of Bessel functions. Soviet Math. Dokl., 39:173-178, 1990.

[17] S.L. Woronowicz. Unbounded Elements Affiliated with $C^{*}$-algebras and Non-Compact Quantum Groups. Commun. Math. Phys., 136:399-432, 1991. 